\title{
Validación de la Versión Breve de la Experiences in Close Relationship en Adultos con Pareja del mismo Sexo
}

\author{
Validation of the Short Version of the Experiences in Close Relationship in Adults \\ with a same Sex Partner
}

\author{
Cristóbal Calvilloํㅜ, María del Mar Sánchez-Fuentes ${ }^{2}$, Tesifón Parrón Carreño ${ }^{3}$ y Juan Carlos Sierra ${ }^{4}$
}

\begin{abstract}
Resumen
El apego incluye dos dimensiones (ansiedad por abandono y evitación de la intimidad). Para su evaluación se llevó a cabo la validación de la versión breve de la escala Experiences in Close Relationship (ECR-S) en adultos con pareja del mismo sexo. Se examinó la validez de constructo, la invarianza factorial, el funcionamiento diferencia del ítem, la fiabilidad de consistencia interna y la validez concurrente. Se empleó una muestra de 815 homosexuales y 815 heterosexuales (mitad hombres y mitad mujeres), todos ellos en una relación de pareja. Se confirma la estructura bifactorial de la ECR-S, siendo invariante por orientación sexual y sexo. Sus dos dimensiones presentan una consistencia interna adecuada y sus medidas manifiestan evidencias de validez. La ECR-S constituye una excelente herramienta para evaluar el apego en adultos homosexuales con pareja del mismo sexo.
\end{abstract}

Palabras clave: apego, parejas del mismo sexo, ansiedad, evitación, propiedades psicométricas

\begin{abstract}
Attachment includes two dimensions (anxiety by abandonment and avoidance of intimacy). For its evaluation, the validation of the brief version of the Experiences in Close Relationship (ECR-S) scale was carried out in adults with a same-sex partner. Construct validity, factorial invariance, differential item functioning, reliability of internal consistency and concurrent validity were examined. A sample of 815 homosexuals and 815 heterosexuals (half men and half women) were used, all of them in a couple relationship. The bifactorial structure of the ECR-S is confirmed, being invariant by sexual orientation and sex. Its two dimensions have an adequate internal consistency and their measurements show evidence of validity. The ECR-S constitutes an excellent tool for assessing attachment in homosexual adults with a samesex partner.
\end{abstract}

Keywords: attachment, same-sex relationship, anxiety, avoidance, psychometric properties

\footnotetext{
${ }^{1}$ Máster en Sexología. Doctorando. Centro de Investigación Mente, Cerebro y Comportamiento (CIMCYC), Universidad de Granada. Campus Universitario de Cartuja, s/n. Granada, España. Tel.: +34638128788. Correo: ccalvillo@ correo.ugr.es

${ }^{2}$ Doctora en Psicología. Profesora de Universidad. Facultad de Ciencias Humanas y Sociales, Departamento de Ciencias Sociales, Universidad de la Costa, Calle 58, \# 55 - 66. Barranquilla, Colombia. Tel.: +573004674791. Correo: msanchez26@cuc.edu.co

${ }^{3}$ Doctor en Medicina y Cirugía. Profesor de Universidad. Edificio Ciencias de la Salud, Departamento de Enfermería, Fisioterapia y Medicina, Universidad de Almería. Carretera Sacramento, s/n, Almería, España. Tel.: +34950214604. Correo: tpc468@ual.es

${ }^{4}$ Doctor en Psicología. Catedrático de Universidad. Centro de Investigación Mente, Cerebro y Comportamiento (CIMCYC), Universidad de Granada. Facultad de Psicología. Campus Universitario de Cartuja, s/n. 18011 Granada, España. Tel.: +34958243750. Correo: jcsierra@ugr.es (correspondencia) 


\section{Introducción}

El apego se refiere al proceso de vinculación emocional originado en la infancia de los seres humanos con sus primeros cuidadores (Bowlby, 1973, 1982; Gómez-Zapiain, 2009). En la edad adulta juega un papel crucial en las relaciones con los iguales, provocando la tendencia a buscar y mantener la proximidad, así como el contacto con determinadas personas que nos proveerán seguridad psicológica y/o física (Sperling \& Berman, 1994). La forma en como las personas buscan y mantienen esa proximidad está influenciada por los modelos internos, creados desde la infancia, que se expresan en los diferentes estilos de apego existentes. Todo esto hace que el apego sea un factor importante para el entendimiento del desarrollo del ser humano (Román, Hodges, Palacios, Moreno, \& Hillman, 2018).

El apego está compuesto de dos dimensiones básicas: ansiedad por abandono y evitación de la intimidad (Brennan, Clark, \& Shaver, 1998). La primera se refiere a un excesivo miedo al rechazo y abandono por parte de la figura de apego, caracterizándose por una visión negativa hacia uno mismo y positiva hacia los demás. La segunda dimensión consiste en la incomodidad con la proximidad e intimidad, siendo caracterizada por una visión positiva hacia uno mismo y negativa hacia los demás (Mikulincer, 2006). Dependiendo del nivel de ansiedad y evitación, el apego se categoriza en diferentes estilos: seguro, ansioso-ambivalente y evitativo (Hazan \& Shaver, 1987). Las personas con estilo seguro tienden a tener una sana proximidad en las relaciones íntimas, caracterizándose por un nivel bajo de ansiedad y evitación. Las personas con un estilo de apego ansioso-ambivalente presentan miedo a ser abandonados o rechazados por su figura de apego (Bartholomew \& Horowitz, 1991), se enamoran de forma rápida e indiscriminada, y utilizan las relaciones sexuales para obtener cercanía emocional de su pareja (Guzman, Rovella, \& Rivarola, 2012). Este estilo se caracteriza por alto nivel de ansiedad y bajo nivel de evitación. Por último, las personas con un estilo evitativo se interesan poco por las relaciones íntimas y tienen poca disposición a mantener relaciones a largo plazo. Pueden tener relaciones sexuales satisfactorias, pero se muestran incapaces de intimar de manera emocional. El apego evitativo se conforma por baja ansiedad y alta evitación.

El estudio de la Teoría del Apego en las relaciones adultas ha promovido numerosas investigaciones referentes al amor y a las relaciones de pareja (Gómez-Zapiain, 2009; Gómez-Zapiain, Ortiz, \& Gómez-Lope, 2012), esto es debido a que "el amor puede ser considerado como la expresión del vínculo de apego en las relaciones afectivas entre adultos" (Hazan \& Shaver, 1987, p. 511). Existen diferencias en los estilos de apego según el sexo (Scharfe, 2017). Los hombres tienden a presentar un estilo más evitativo que las mujeres (Antičević, Britvić, \& Jokić-Begić, 2018; Del Giudice, 2011, 2019), mientras que estas lo suelen presentar más ansioso (Dunkel, Lukaszewski, \& Chua, 2016). Sin embargo, estas diferencias se ha examinado mayoritariamente en población heterosexual. Los estudios realizados con población homosexual muestran resultados más dispares. Craft, Serovich, Mckenry y Lim (2008) no encuentran diferencias en los estilos de apego entre hombres y mujeres con orientación homosexual, mientras que Guzmán et al. (2016) señalan que los hombres homosexuales presentan un estilo de apego más evitativo y ansioso que las mujeres; por su parte, Ridge y Feeney (1998) informaron que las mujeres homosexuales presentaban un estilo más evitativo que los hombres. A su vez, los estudios que han comparado los estilos de apego en función de la orientación sexual también muestran resultados contradictorios. Mientras en algunos no encuentran diferencias entre los estilos de apego por orientación sexual (Farr, Forssell, \& Patterson, 2010; Ridge \& Feeney, 1998), otros concluyen que las parejas heterosexuales manifiestan un estilo de apego más evitativo que las parejas del mismo sexo (Domingue \& Mollen, 2009). Por tanto, son necesarias más investigaciones que examinen las diferencias en los estilos de apego en población homosexual.

En relación con las medidas que evalúan el apego, la escala Experiences in Close Relationship (Brennan et al., 1998) es una herramienta válida para el estudio del apego en función de sus dimensiones, tanto a nivel clínico como de investigación (Bartholomew \& Shaver, 
Tabla 1. Variables sociodemográficas por sexo y orientación sexual

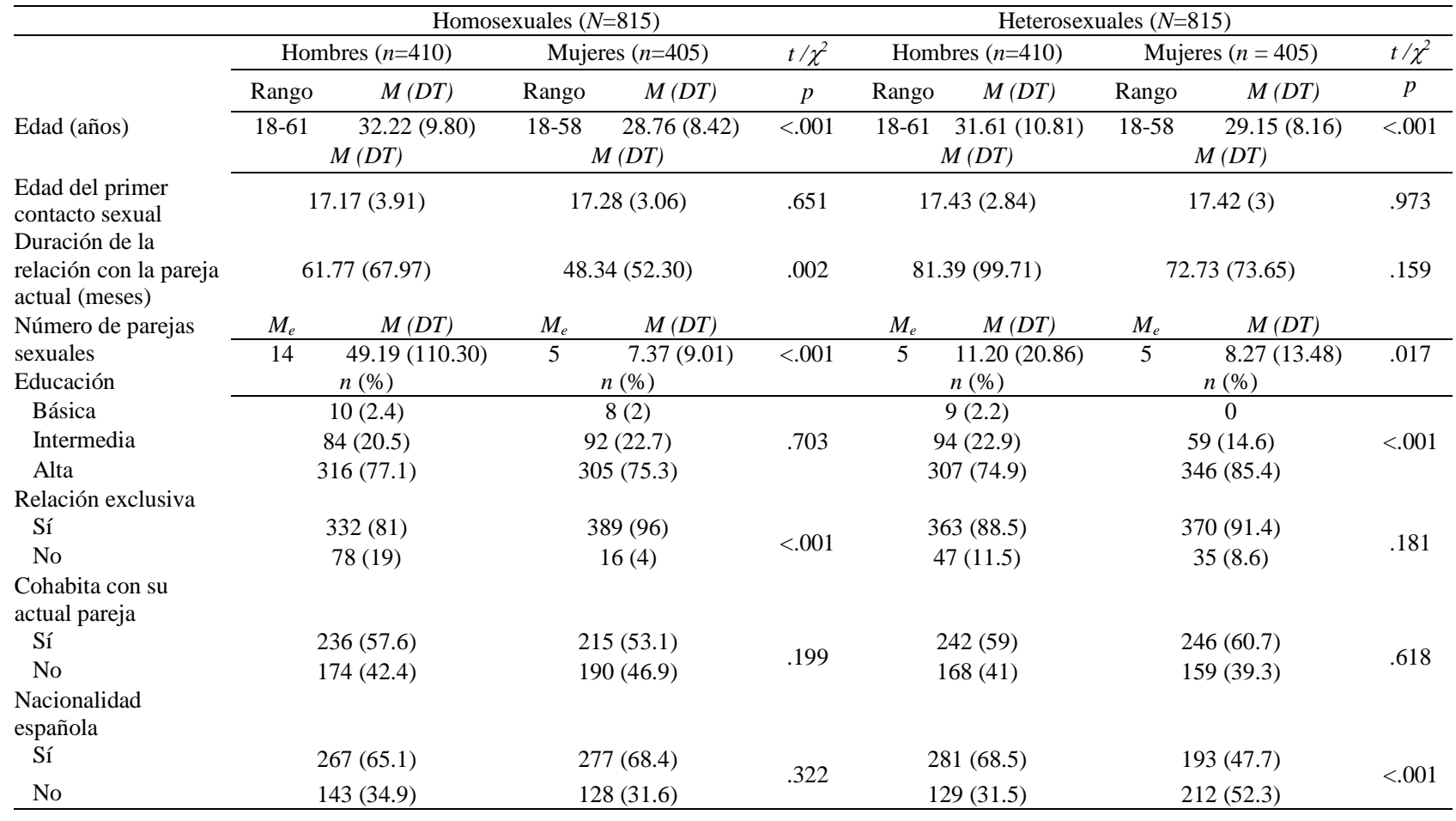

1998; Fraley \& Waller, 1998; Griffin \& Bartholomew, 1994; Martínez \& Santelices, 2005). Dicha escala presenta adecuadas propiedades psicométricas para evaluar el apego adulto en población española (Alonso-Arbiol, Balluerka, \& Shaver, 2007; Yárnoz-Yaben \& Comino, 2011). Se ha desarrollado una versión breve de 12 ítems (ECR-S; Wei, Russell, Mallinckrodt, \& Vogel, 2007), que no ha sido objeto de estudio psicométrico en población homosexual, limitación que presentan muchos instrumentos de evaluación en el área de la sexualidad humana (Calvillo, Sánchez-Fuentes, \& Sierra, 2018). Así pues, el objetivo de este estudio es validar la Experiences in Close RelationshipShort Form (ECR-S; Wei et al., 2017) en adultos hispanohablantes con pareja del mismo sexo. Los objetivos específicos son: (a) examinar si la estructura factorial de la ECR-S es invariante por orientación sexual; (b) analizar el funcionamiento diferencial de sus ítems por orientación sexual; (c) examinar su fiabilidad de consistencia interna; y (d) aportar evidencias de validez de sus medidas, correlacionándolas con la satisfacción con la relación de pareja y el ajuste diádico. En base a estudios previos se plantean las siguientes hipótesis: (H1) se espera que la estructura factorial del ECR-S sea invariante por sexo (Lafontaine et al., 2016) y orientación sexual;
(H2) las puntuaciones en ansiedad y evitación correlacionarán en sentido negativo con la satisfacción con la relación de pareja (Mark, Vowels, \& Murray, 2018) y el ajuste diádico (Lafontaine et al., 2016; Pepping \& Halford, 2012; Riggs, Cusimano, \& Benson, 2011); y (H3) se espera que los hombres presenten mayor evitación que las mujeres, y que estas manifiesten mayor ansiedad que ellos (Del Giudice, 2011, 2019).

\section{Método}

\section{Participantes}

Mediante un muestreo no probabilístico por conveniencia se seleccionaron 815 participantes homosexuales (410 hombres y 405 mujeres) que cumplían con los siguientes criterios de inclusión: (a) origen español o hispanoamericano; (b) idioma español como lengua materna; (c) mayor de edad; (d) orientación homosexual; (e) cisgénero; y (f) estar en una relación de pareja con otra persona cisgénero y del mismo sexo con una duración de tres meses o más. Además, se contó con otra muestra incidental compuesta por 815 participantes heterosexuales (410 hombres y 405 mujeres) con características similares a la muestra homosexual. Así pues, la muestra total estuvo compuesta por 1,630 participantes. Véase la Tabla 1. 


\section{Instrumentos}

\section{Cuestionario Sociodemográfico y de la Historia}

Sexual. Recopila información sobre sexo, edad, nacionalidad, pareja, sexo de la pareja, edad de la pareja, duración de la relación de pareja, tipo de relación (exclusiva o no), cohabitación con la pareja, relaciones sexuales con la pareja actual, edad de la primera experiencia sexual (anal, oral o vaginal) y número de parejas sexuales.

Escala Kinsey (Kinsey, Pomeroy, \& Martin, 1998). Evalúa la orientación sexual a través de ocho opciones desde exclusivamente heterosexual hasta exclusivamente homosexual. Se incluyó una octava opción de asexualidad. Para la muestra homosexual se seleccionaron los participantes que marcaron la opción exclusivamente homosexual, mientras que para la heterosexual los que señalaron la opción exclusivamente heterosexual.

Versión española de la Experiences in Close Relationship Short-Form (ECR-S; Wei et al., 2007). Esta escala evalúa el apego adulto dentro del contexto de las relaciones de pareja por medio de 12 ítems (seis de Evitación y seis de Ansiedad) contestados en una escala de respuesta tipo Likert de siete puntos desde 1 (totalmente en desacuerdo) a 7 (totalmente de acuerdo). A mayor puntuación mayor ansiedad o evitación. De la versión extensa en español de Alonso-Arbiol et al. (2007) se extrajeron los 12 ítems que componen la ECR-S.

Versión española del Global Measure of Relationship Satisfaction (GMREL; SánchezFuentes, Santos-Iglesias, Byers, \& Sierra, 2015). Evalúa la satisfacción general con la relación de pareja por medio de cinco ítems contestados en una escala Likert de siete puntos cada una, mayor puntuación indica mayor satisfacción con la relación. La versión española presenta adecuadas propiedades psicométricas (Sánchez-Fuentes et al., 2015). En el presente estudio los coeficientes de fiabilidad de consistencia interna fueron $.94 \mathrm{en}$ hombres y .92 en mujeres homosexuales.

Versión española de la versión breve del Dyadic Adjustment Scale (DAS; Santos-Iglesias, VallejoMedina, \& Sierra, 2009). Compuesta por 13 ítems que evalúan el ajuste diádico a través de tres dimensiones (Satisfacción, Consenso y Cohesión). La escala utiliza opciones de respuesta de seis y cinco alternativas, dependiendo de los ítems. Puntuaciones más altas indican un mayor ajuste. La escala presenta adecuada fiabilidad y validez (Santos-Iglesias et al., 2009). En el presente estudio la fiabilidad de consistencia interna fue de .79, tanto en hombres como en mujeres homosexuales.

\section{Procedimiento}

La aplicación de las escalas se realizó en formato de papel y lápiz, y formato en línea. Ambos métodos se emplean en la validación de escalas relacionadas con la salud sexual (ArcosRomero \& Sierra, 2019; Sánchez-Fuentes, Moyano, Granados, \& Sierra, 2019) y son igual de fiables y válidos (Sierra, Moyano, VallejoMedina, \& Gómez-Berrocal, 2018). Los participantes que completaron los cuestionarios en formato papel fueron contactados, a través del método bola de nieve, en lugares públicos y de ocio, así como, por asociaciones de lesbianas, gays, bisexuales y transexuales (LGBT). Para ello, los evaluadores asistían a actividades realizadas por asociaciones LGBT, en las que se informaba sobre la investigación y se les invitaba a participar de manera voluntaria. Los participantes interesados recibían la batería de autoinformes junto con un sobre, para que una vez cumplimentados fueran devueltos en sobre cerrado, con el fin de asegurar el anonimato y confidencialidad. También se contactó con asociaciones a través de correo electrónico, a las que se informó sobre el objetivo principal del estudio y del procedimiento. A las que accedieron a participar se les enviaban las encuestas en papel y sobres, que eran devueltos a los investigadores una vez contestados. Para la versión online, esta fue diseñada en Limesurvey ${ }^{\circledR}$, se distribuyó a través de plataformas virtuales (Facebook $^{\circledR}$, Twitter $^{\circledR}$, grupos de Whatsapp $^{\circledR}$ y correo electrónico), controlando la dirección de IP en las respuestas. Los participantes completaron los cuestionarios de forma voluntaria, garantizándose en todo momento su anonimato, así como la confidencialidad de los datos obtenidos. Antes de completar los cuestionarios, se presentaba el consentimiento informado que incluía una descripción del objetivo del estudio, e información acerca de la confidencialidad y privacidad de los datos. Entre los participantes con orientación homosexual, 41 (10\%) hombres completaron la encuesta en formato papel y lápiz, 

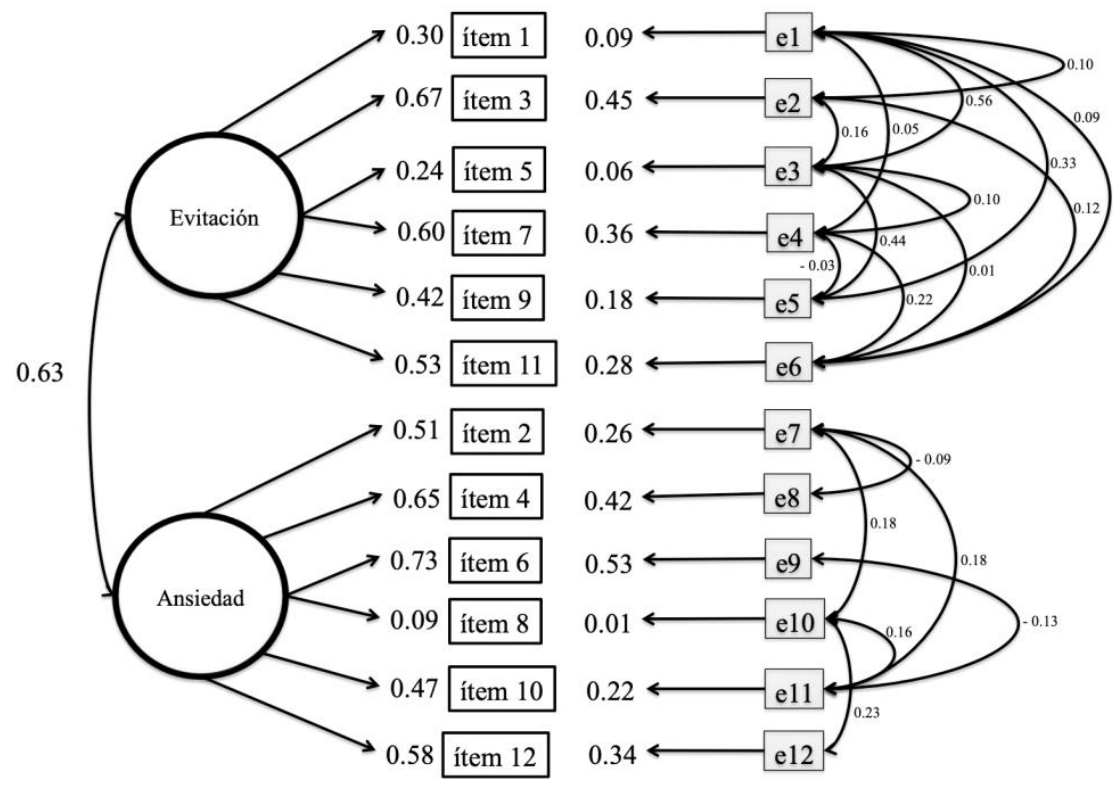

Nota. Muestra total ( $\mathrm{N}=1.630 ; 820$ hombres y 810 mujeres)
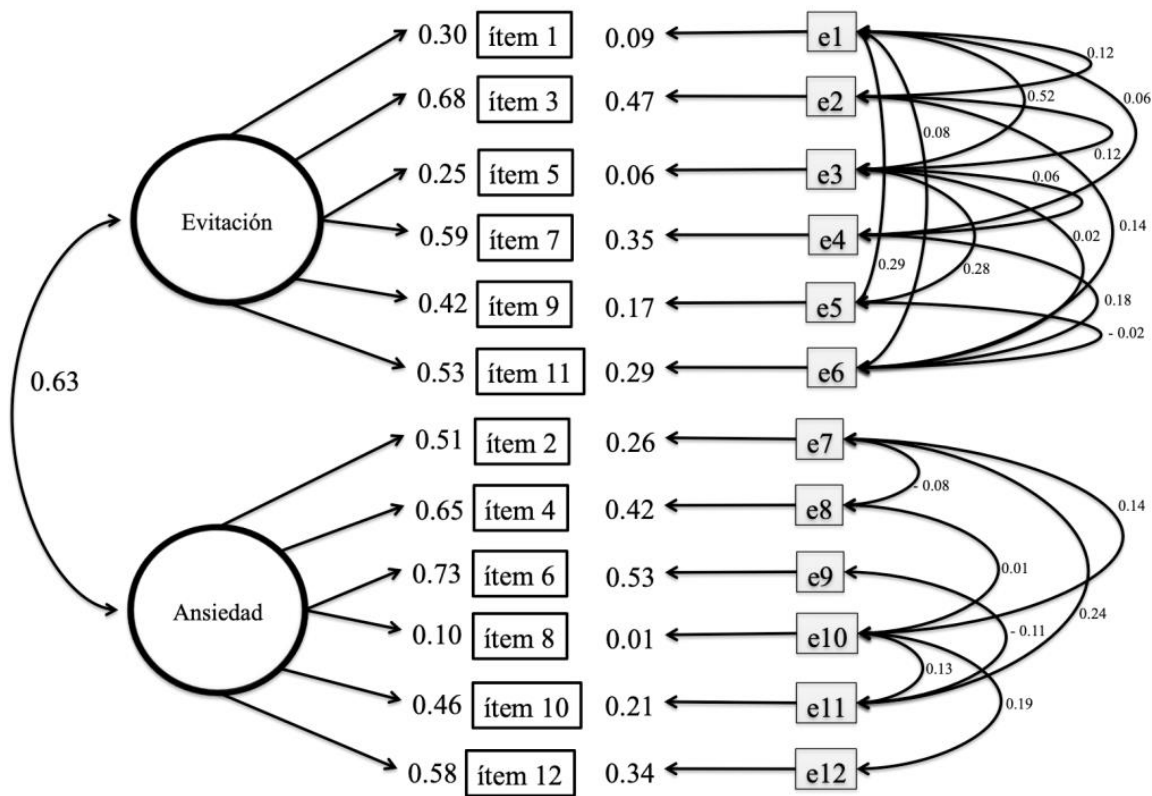

Nota. Muestra total ( $N=1.630 ; 815$ heterosexuales y 815 homosexuales)

Figura 1. Análisis Factorial Confirmatorio (AFC) en hombres y mujeres, y en heterosexuales y homosexuales

y $369(90 \%)$ lo hicieron de manera online; 25 (6.25\%) mujeres respondieron en papel y lápiz, y $380(93.8 \%)$ en formato online. Con respecto a los participantes heterosexuales, 125 (30.5\%) hombres contestaron en formato papel y lápiz, mientras que $285(69.5 \%)$ lo hicieron en formato online; 13 (3.2\%) mujeres contestaron en papel y lápiz, y 392 (96.8\%) de manera online.

\section{Análisis de datos}

Se realizó un Análisis Factorial Confirmatorio (AFC) con el software $I B M^{\circledR}$ SPSS AMOS. En un primer análisis se definieron los grupos hombres y mujeres; para el segundo, los grupos fueron homosexuales y heterosexuales. Se utilizó el procedimiento de máxima verosimilitud (Wang \& Wang, 2012). Para evaluar el ajuste del AFC multigrupo se tuvieron en cuenta los siguientes criterios: un CFI y un TLI > .90 y un RMSEA < .08 (Hu \& Bentler, 1999; Wang \& Wang, 2012), y la diferencia entre los valores del índice comparativo en el CFI (Bentler, 1990). Teniendo en cuenta la jerarquía de los modelos anidados y cada vez más restrictivos, los modelos se compararon entre sí. Asimismo, se consideró que si la diferencia entre dos modelos anidados tiene 
Tabla 2. Valores de los índices de ajuste de los diferentes modelos por sexo y orientación sexual

\begin{tabular}{lccccccccc}
\hline Modelo & RMSEA & $\begin{array}{c}90 \% \text { CI } \\
\text { RMSEA }\end{array}$ & CFI & TLI & GFI & RMSR & $\begin{array}{c}\text { CMIN/ } \\
\text { DF }\end{array}$ & $\chi^{2}$ & $p$ \\
\hline Sexo & & & & & & & & & \\
1. Configural & .041 & $.036-.047$ & .957 & .919 & .974 & .134 & 3.756 & 262.916 & $<.001$ \\
2. Débil & .039 & $.034-.044$ & .955 & .926 & .972 & .147 & 3.508 & 280.629 & $<.001$ \\
3. Fuerte & .039 & $.035-.044$ & .953 & .925 & .971 & .154 & 3.538 & 293.674 & $<.001$ \\
4. Estricta & .039 & $.034-.043$ & .948 & .928 & .967 & .168 & 3.457 & 328.424 & $<.001$ \\
Orientación sexual & & & & & & & & & \\
1. Configural & .041 & $.036-.047$ & .958 & .919 & .974 & .136 & 3.796 & 258.119 & $<.001$ \\
2. Débil & .040 & $.035-.045$ & .956 & .925 & .972 & .145 & 3.569 & 278.405 & $<.001$ \\
3. Fuerte & .039 & $.034-.044$ & .955 & .927 & .972 & .158 & 3.489 & 282.595 & $<.001$ \\
4. Estricta & .038 & $.033-.043$ & .952 & .932 & .969 & .159 & 3.329 & 309.571 & $<.001$ \\
\hline
\end{tabular}

Nota. RMSEA: Root Mean Square Error of Approximation; CI: Confidence Interval; CFI: Comparative Fit Index; TLI: Tucker-

Lewis Index; GFI: Goodness of Fit Index; RMSR; Root Mean Square Residual; CMIN/DF: Chi Square per Degree of Freedom.

un valor en el CFI superior a .01 a favor del modelo con menos restricciones, se rechazaría el modelo con más restricciones (Cheung \& Rensvold, 2002). Se examinaron las medidas de invarianza que analizaban la relación entre las variables observables y las variables latentes (Milfont \& Fischer, 2010). Para el cálculo del funcionamiento diferencial del ítem (DIF) se empleó el software EASY-DIF (González, Padilla, Hidalgo, Gómez-Benito, \& Benítez, 2011), que estima el funcionamiento diferencial en ítems politómicos utilizando un procedimiento de estandarización (Dorans \& Holland, 1993; Zwick, Donoghue, \& Grima, 1993) y la media del estadístico de Mantel (1963).

Mediante los coeficientes omega (McDonald, 1999; Ventura-León, 2018) y alfa ordinal (Zumbo, Gadermann, \& Zeisser, 2007) se estimó la fiabilidad de consistencia interna. Para calcular el omega se utilizó el paquete MBESS® (Paquete de R, Versión 4.2.0; Kelley, 2018), y para el alfa ordinal, el paquete userfriendlyscience ${ }^{\circledR}$ (Paquete de R, Versión 0.7.2; Peters, 2018). Ambos paquetes forman parte del programa $R \circledR$ (Versión 3.4.4; $\mathrm{R}$ Core Team, 2016), con su interfaz integrado RStudio ${ }^{\circledR}$ (Versión 1.1.447; RStudio Team, 2018). Se consideró el omega jerárquico, que da la proporción de varianza de las puntuaciones de cada escala explicada por un factor general (McDonald, 1999; Zinbarg, Revelle, \& Yovel, 2007).

\section{Resultados}

\section{Análisis Factorial Confirmatorio (AFC)}

$\mathrm{Se}$ establecieron dos modelos con las dimensiones Evitación y Ansiedad: sexo y orientación sexual (Figura 1). Los resultados mostraron que el modelo bidimensional de la escala ECR-S presentó covarianzas entre sus variables observables en sus diferente análisis multigrupales. Se evidenció invarianza estricta en ambos modelos gracias a los valores relativos al CFI que permitieron aceptar la equivalencia factorial para los distintos niveles de ajuste. En la invarianza por sexo, se obtuvo un RMSEA de .039 con su I.C. del $90 \%$ entre .34 y .43 , y un CFI de .948 para su nivel de invarianza estricta. En relación a la invarianza por orientación sexual, se obtuvo un RMSEA de .038 con su I.C. del $90 \%$ entre .033 y .043 , y un CFI de .952 para su nivel estricto (Tabla 2).

En cuanto al DIF, al comparar por orientación sexual, es decir, hombres heterosexuales con hombres homosexuales y mujeres heterosexuales con mujeres homosexuales, en hombres se evidenció DIF en los ítems 3 y 7, ambos pertenecientes a Evitación, y en los ítems 2, 4, 10 y 12 en Ansiedad. En mujeres, solamente hubo DIF en el ítem 8 de Ansiedad. Posteriormente, se comparó por sexo en los participantes homosexuales, encontrándose DIF en los ítems 10 y 12, ambos pertenecientes a Ansiedad (Tabla 3). 
Tabla 3. Funcionamiento diferencial del ítem (DIF) por orientación sexual y por sexo en homosexuales

\begin{tabular}{|c|c|c|c|c|c|c|c|c|c|}
\hline \multirow{2}{*}{$\begin{array}{l}\text { Variables } \\
\text { Evitación }\end{array}$} & \multicolumn{3}{|c|}{$\begin{array}{c}\text { Hombres } \\
\text { (Heterosexuales-Homosexuales) }\end{array}$} & \multicolumn{3}{|c|}{$\begin{array}{c}\text { Mujeres } \\
\text { (Heterosexuales-Homosexuales) }\end{array}$} & \multicolumn{3}{|c|}{$\begin{array}{c}\text { Homosexuales } \\
\text { (Hombres-Mujeres) }\end{array}$} \\
\hline & $\chi^{2}$ & $p$ & $S P D$ & $\chi^{2}$ & $p$ & $S P D$ & $\chi^{2}$ & $p$ & $S P D$ \\
\hline Ítem 1 & 0.48 & .487 & .022 & 0.26 & .608 & .040 & 0.80 & .370 & .035 \\
\hline Ítem 3 & 5.89 & .015 & .142 & 0.64 & .423 & -.068 & 0.65 & .420 & -.052 \\
\hline Ítem 5 & 1.36 & .242 & .059 & 2.76 & .096 & -.081 & 0.44 & .508 & -.061 \\
\hline Ítem 7 & 5.57 & .018 & -.225 & 0.09 & .769 & -.041 & 0.03 & .857 & .025 \\
\hline Ítem 9 & 0.07 & .798 & .016 & 2.92 & .087 & .089 & 0.62 & .429 & -.048 \\
\hline Ítem 11 & 0.04 & .832 & -.014 & 1.05 & .306 & .061 & 2.23 & .135 & .101 \\
\hline Ansiedad & & & & & & & & & \\
\hline Ítem 2 & 6.45 & .011 & .245 & 0.18 & .667 & .025 & 0.32 & .569 & .059 \\
\hline Ítem 4 & 4.42 & .035 & -.259 & 0.12 & .725 & -.025 & 2.81 & .093 & -.151 \\
\hline Ítem 6 & 0.46 & .498 & -.099 & 0.15 & .703 & .022 & 0.17 & .677 & -.041 \\
\hline Ítem 8 & 3.25 & .071 & .197 & 8.62 & .003 & .426 & 0.55 & .458 & .091 \\
\hline Ítem 10 & 14.14 & .000 & .436 & 3.50 & .061 & -.220 & 3.88 & .048 & -.188 \\
\hline Ítem 12 & 8.60 & .003 & -.52 & 2.74 & .097 & -.228 & 5.22 & .022 & .230 \\
\hline
\end{tabular}

Tabla 4. Correlaciones entre los factores Evitación y Ansiedad de la versión española del ECR-S con la medida de GMREL, la escala de Ajuste diádico y sus dimensiones (Consenso, Cohesión y Satisfacción) en hombres y mujeres homosexuales

\begin{tabular}{lccccccc}
\hline & 1 & 2 & 3 & 4 & 5 & 6 & 7 \\
\hline 1. Evitación & - & $.25^{* *}$ & $-.43^{* *}$ & $-.56^{* *}$ & $-.38^{* *}$ & $-.40^{* *}$ & $-.57^{* *}$ \\
2. Ansiedad & $.42^{* *}$ & - & $-.28^{* *}$ & $-.43^{* *}$ & $-.39^{* *}$ & $-.36^{* *}$ & $-.36^{* *}$ \\
3. GMREL & $-.36^{* *}$ & $-.29^{* *}$ & - & $.56^{* *}$ & $.47^{* *}$ & $.34^{* *}$ & $.53^{* *}$ \\
4. Ajuste diádico & $-.52^{* *}$ & $-.41^{* *}$ & $.59^{* *}$ & - & $.86^{* *}$ & $.72^{* *}$ & $.80^{* *}$ \\
5. Dominio Consenso & $-.32^{* *}$ & $-.32^{* *}$ & $.40^{* *}$ & $.81^{* *}$ & - & $.44^{* *}$ & $.52^{* *}$ \\
6. Dominio Cohesión & $-.34 * *$ & $-.26^{* *}$ & $.39^{* *}$ & $.70^{* *}$ & $.36^{* *}$ & - & $.40^{* *}$ \\
7. Dominio Satisfacción & $-.53^{* *}$ & $-.36^{* *}$ & $.58^{* *}$ & $.79 * *$ & $.42^{* *}$ & $.39 * *$ & - \\
\hline
\end{tabular}

Nota. GMREL: Global Measure of Relationship Satisfaction. ** $p<.01$. Encima de la diagonal, las correlaciones de los hombres; debajo de la diagonal, las de las mujeres.

\section{Fiabilidad}

Para la dimensión Evitación, en hombres homosexuales, se obtuvo un omega de .70 y un alfa ordinal de .85; en mujeres homosexuales, los coeficientes fueron .78 y .87 , respectivamente. En el caso de la dimensión Ansiedad, en hombres homosexuales, el coeficiente omega fue $.70 \mathrm{y}$ el alfa ordinal .74; en mujeres homosexuales, se obtuvieron valores de .68 y .74 , respectivamente.

\section{Evidencias de validez}

Mediante correlaciones de Pearson se examinaron las hipótesis sobre la validez. Debido al gran tamaño muestral se empleó un alfa conservador de $p<.01$. En general, los resultados confirmaron validez para ambos constructos de la escala. Tanto en hombres como mujeres homosexuales, las dimensiones Evitación y Ansiedad se asociaron de forma negativa con la medida de satisfacción con la relación y de ajuste diádico, así como con las dimensiones de este (Cohesión, Consenso y Satisfacción). Las dos dimensiones del apego correlacionaron entre sí en sentido positivo (Tabla 4).
Para determinar si la magnitud de las correlaciones entre cada par de medidas difería significativamente entre hombres y mujeres, se realizó una serie de transformaciones de Fisher $r$ a puntajes $z$ para evaluar la importancia de las diferencias entre cada par de coeficiente de correlación. Posteriormente, se usó la corrección de Bonferroni para evitar el error tipo I inflado. Se obtuvo un coeficiente de alfa de Cronbach de .002 y de las 21 comparaciones que hubo, no se hallaron correlaciones estadísticamente diferentes en hombres y mujeres homosexuales. Por tanto, los patrones de las correlaciones entre las medidas del ECR-S, satisfacción con la relación y la medida de ajuste diádico son similares para hombres y mujeres homosexuales.

\section{Niveles de ansiedad y evitación en hombres y mujeres homosexuales}

Se comparan por sexo las puntuaciones en Evitación y Ansiedad de la versión española del ECR-S en la muestra homosexual. Se encontraron diferencias estadísticamente significativas solamente en Evitación $(U=71140 ; p<.001)$, 
siendo los hombres los que obtuvieron mayor puntuación (Evitación, $M_{\text {hombres }}=12.62, D T=6.38$ y $M_{\text {mujeres }}=11.24, D T=6.04$ ), no ocurriendo lo mismo con Ansiedad $(U=79347.50 ; \quad p=.273$; $M_{\text {hombres }}=19.86, \quad D T=7.45 \quad$ y $\quad M_{\text {mujeres }}=19.22$, $D T=7.53)$.

\section{Discusión}

Se examinaron las propiedades psicométricas de la versión española de la escala Experiences in Close Relationship (ECR-S) en personas adultas hispanohablantes con pareja del mismo sexo. Tras los resultados obtenidos se comprueba que la versión española del ECR-S es un instrumento fiable y válido para evaluar el apego a través de las dimensiones de ansiedad y evitación en personas adultas con pareja del mismo sexo.

Las dimensiones Evitación y Ansiedad que forman la versión española del ECR-S constituyen dos medidas diferentes, aunque relativamente relacionadas, tal como se evidenció también en el estudio de Sibley y Liu (2004) con la escala de 36 ítems. Se analizó la invarianza factorial por sexo y por orientación sexual, mostrando ambos modelos invarianza estricta, confirmándose de este modo la hipótesis 1, es decir, que la estructura de la escala no varía en función del sexo ni de la orientación sexual. En relación al análisis del funcionamiento diferencial de los ítems por orientación sexual, aunque se mostró mayor presencia de DIF en el grupo de hombres que en el de mujeres, esto no supone una amenaza para la validez del instrumento debido a que el análisis factorial confirmatorio multigrupal reflejó una invarianza estricta con excelentes valores de ajuste, tanto por sexo como por orientación sexual, lo cual indica una equivalencia de medida entre los grupos analizados. Por otro lado, en el análisis por sexo en el grupo con orientación homosexual, se evidenció DIF solamente en dos ítems. Esto indica que, de manera general, hombres y mujeres homosexuales no presentan diferente probabilidad de responder a la escala. Sin embargo, para futuras investigaciones se recomienda la eliminación de los dos ítem al momento de comparar el apego entre hombres y mujeres homosexuales. En cuanto a la fiabilidad, las dos dimensiones del ECR-S alcanzaron coeficientes de fiabilidad de consistencia interna aceptables (Carretero-Dios \& Pérez, 2005). Es fundamental disponer de medidas invariantes en cuanto a la orientación sexual (Calvillo et al., 2018), tal como se demostró por ejemplo en la experiencia subjetiva del orgasmo (Arcos-Romero \& Sierra, 2019)

En cuanto a las evidencias de validez, se obtuvieron correlaciones negativas de ambas dimensiones del apego (Evitación y Ansiedad) con el ajuste diádico y la satisfacción con la relación de pareja, confirmándose de este modo la hipótesis 2. Otras investigaciones habían demostrado que un apego evitativo o ansioso se relaciona con baja satisfacción con la relación de pareja (Collins \& Read, 1990; Li \& Chan, 2012; Mikulincer \& Shaver, 2016; Schachner \& Shaver, 2004), lo que podría deberse a las dificultades en las estrategias de regulación del afecto, propias de individuos con apego inseguro (Hocking, Simons, Simons, \& Freeman, 2018; Li \& Chan, 2012). Las personas con apego seguro presentarían buenas estrategias emocionales para relacionarse sanamente con su pareja, independientemente de la orientación sexual (MacIntosh, Reissing, \& Andruff, 2010).

En este estudio, las puntuaciones obtenidas por hombres y mujeres con pareja del mismo sexo fueron relativamente bajas en evitación y en ansiedad. Se podría decir que presentan un apego de tipo seguro. Se encontraron diferencias significativas por sexo únicamente en evitación, siendo los hombres los que puntuaron más alto. El hecho de que los hombres hayan presentado más evitación que las mujeres coincide con los trabajos de Del Giudice (2011, 2019). Que los hombres homosexuales presenten más evitación que las mujeres podría ser debido a factores culturales y estereotipados relacionados con el rol de género, de acuerdo con la teoría de los guiones sexuales (Simon \& Gagnon, 1986). Estos guiones tradicionales son diferentes para hombres y mujeres, caracterizándose en los hombres por mantener relaciones sexuales sin o con poca involucración emocional (Alksnis, Desmarais, \& Wood, 1996; Katz \& Schneider, 2015), lo que coincide con el estilo de apego evitativo. Cabría pensar que los guiones sexuales refuerzan, en cierto grado, los estilos de apego de hombres y mujeres. No obstante, dado que los guiones sexuales se centran en el rol de género de 
personas heterosexuales, se sugiere más investigación al respecto. Por otro lado, la homofobia internalizada pudiera estar influyendo en el hecho de que, en esta muestra, los hombres presenten más evitación que las mujeres. Los apegos inseguros se asocian con la dificultad para aceptar la propia sexualidad (Mohr \& Fassinger, 2003) y la homofobia internalizada (Sherry, 2007; Starks \& Parsons, 2014). Personas homosexuales con apego evitativo tienden a presentar más actitudes homonegativas de manera internalizada y más dificultad para relacionarse de manera íntima con los demás (Wang, Schale, \& Broz, 2010) que las personas seguras. En definitiva, el sexo pudiera jugar un rol importante en la relación de la homofobia internalizada con el apego evitativo, de manera que, en primer lugar, los hombres homosexuales experimentarían más actos de homofobia externa que las mujeres (Balsam, Rothblum, \& Beauchaine, 2005; D'Augelli, Pilkington, \& Hershberger, 2002; Mclaren, 2015) $\mathrm{y}$, en segundo lugar, las muestras de afecto son más restrictivas socialmente en hombres homosexuales que en mujeres (Gonsiorek, 1995; Jellison \& McConnell, 2004). Esto reforzaría la vinculación de la internalización de actitudes homonegativas con el apego evitativo, presentándose más en hombres homosexuales que en mujeres.

Los resultados obtenidos proporcionan información sobre el apego en personas adultas hispanohablantes con pareja del mismo sexo e incorporan evidencia de las propiedades psicométricas de la versión española de la ECR-S para su empleo en personas homosexuales con pareja. Sin embargo, el estudio presenta algunas limitaciones. La muestra tiene poca variabilidad en el apego, es decir, la mayoría de los participantes presentan un apego seguro. Son necesarias investigaciones que examinen las propiedades psicométricas de la versión española de la ECR-S en participantes que reporten variabilidad en el apego (ya sea ansioso, evitativo y/o temeroso). Así también, se requieren investigaciones que comprueben dichas propiedades psicométricas en personas pertenecientes a diferentes grupos dentro del colectivo LGBT, como personas transexuales o intersexuales. A partir de estas investigaciones se podrían elaborar modelos de promoción de la salud para las personas LGBT en donde se tengan en cuenta aspectos individuales y estructurales en relación con la salud (Sevilla Rodríguez, Aparicio García, \& Limiñana Gras, 2019).

\section{Referencias}

Alksnis, C., Desmarais, S., \& Wood, E. (1996). Gender differences in scripts for different types of dates. Sex Roles, 34(5-6), 321-336. doi:10.1007/BF01547805

Alonso-Arbiol, I., Balluerka, N., \& Shaver, P. R. (2007). A Spanish version of the Experiences in Close Relationships (ECR) adult attachment questionnaire. Personal Relationships, 14(1), 45-63. doi:10.1111/j.1475-6811.2006.00141.x

Antičević, V., Britvić, D., \& Jokić-Begić, N. (2018). Attachment styles and sexuality: The contribution of gender, age and relationship status. Socijalna Psihijatrija, 46(2), 125-141. doi:10.24869/spsih.2018.125

Arcos-Romero, A. I., \& Sierra, J. C. (2019). Factorial invariance, differential item functioning, and norms of the Orgasm Rating Scale. International Journal of Clinical and Health Psychology, 19(1), 57-66. doi:10.1016/j.ijchp.2018.11.001

Balsam, K. F., Rothblum, E. D., \& Beauchaine, T. P. (2005). Victimization over the life span: A comparison of lesbian, gay, bisexual, and heterosexual siblings. Journal of Consulting and Clinical Psychology, 73(3), 477-487. doi:10.1037/0022-006X.73.3.477

Bartholomew, K., \& Horowitz, L. M. (1991). Attachment styles among young adults: A test of a four-category model. Journal of Personality and Social Psychology, 61(2), 226-244. doi:10.1037/0022-3514.61.2.226

Bartholomew, K., \& Shaver, P. R. (1998). Methods of assessing adult attachment: Do they converge? En J.A. Simpson \& W.S. Rholes (Eds.), Attachment theory and close relationships (pp. 25-45). Nueva York, NY: Guilford Press.

Bentler, P. M. (1990). Comparative fit indexes in structural models. Psychological Bulletin, 107(2), 238-246.

doi:10.1037/0033-2909.107.2.238 
Bowlby, J. (1973). Attachment and loss. Separation: Anxiety and anger (Vol. 2). Nueva York, NY: Basic Books.

Bowlby, J. (1982). Attachment and loss ( $2^{\mathrm{a}}$ ed., Vol. 1). Nueva York, NY: Basic Books.

Brennan, K. A., Clark, C. L., \& Shaver, P. R. (1998). Self-report measurement of adult attachment: An integrative overview. En J. A. Simpson y W. S. Rholes (Eds.), Attachment theory and close relationships (pp. 46-76). Nueva York, NY: Guilford Press.

Calvillo, C., Sánchez-Fuentes, M. M., \& Sierra, J. C. (2018). Revisión sistemática sobre la satisfacción sexual en parejas del mismo sexo. Revista Iberoamericana de Psicología y Salud, 9(2), 115-136. doi:10.23923/j.rips.2018.02.018

Carretero-Dios, H., \& Pérez, C. (2005). Normas para el desarrollo y revisión de estudios instrumentales. International Journal of Clinical and Health Psychology, 5(3), 521551.

Cheung, G. W., \& Rensvold, R. B. (2002). Evaluating goodness-of-fit indexes for testing measurement invariance. Structural Equation Modeling: A Multidisciplinary Journal, 9(2), 233-255. doi:10.1207/S15328007SEM0902_5

Collins, N. L., \& Read, S. J. (1990). Adult attachment, working models, and relationship quality in dating couples. Journal of Personality and Social Psychology, 58(4), 644-663. doi:10.1037/0022-3514.58.4.644

Craft, S. M., Serovich, J. M., McKenry, P. C., \& Lim, J.-Y. (2008). Stress, attachment style, and partner violence among same-sex couples. Journal of GLBT Family Studies, 4(1), 57-73. doi:10.1080/15504280802084456

D'Augelli, A. R., Pilkington, N. W., \& Hershberger, S. L. (2002). Incidence and mental health impact of sexual orientation victimization of lesbian, gay, and bisexual youths in high school. School Psychology Quarterly, 17(2), 148-167. doi:10.1521/scpq.17.2.148.20854

Del Giudice, M. (2011). Sex differences in romantic attachment: A meta-analysis. Personality and Social Psychology Bulletin, 37(2), 193-214. doi:10.1177/0146167210392789
Del Giudice, M. (2019). Sex differences in attachment styles. Current Opinion in Psychology, 25, 1-5. doi:10.1016/j.copsyc.2018.02.004

Domingue, R., \& Mollen, D. (2009). Attachment and conflict communication in adult romantic relationships. Journal of Social and Personal Relationships, 26(5), 678-696. doi:10.1177/0265407509347932

Dorans, N. J., \& Holland, P. W. (1993). DIF detection and description: Mantel-Haenszel and standardization. En P. W. Holland y H. Wainer (Eds.), Differential item functioning (pp. 35-66). Hillsdale, NJ: Lawrence Erlbaum Associates.

Dunkel, C. S., Lukaszewski, A. W., \& Chua, K. (2016). The relationship between sex, life history strategy, and adult romantic attachment style. Personality and Individual Differences, 98, 176-178. doi:10.1016/j.paid.2016.04.040

Farr, R. H., Forssell, S. L., \& Patterson, C. J. (2010). Gay, lesbian, and heterosexual adoptive parents: Couple and relationship issues. Journal of GLBT Family Studies, 6(2), 199-213. doi:10.1080/15504281003705436

Fraley, R. C., \& Waller, N. G. (1998). Adult attachment patterns: A test of the typological model. En J. A. Simpson \& W. S. Rholes (Eds.), Attachment theory and close relationships (pp. 77-114). Nueva York, NY: Guilford Press.

Gómez-Zapiain, J. (2009). Apego y sexualidad. Madrid: Alianza Editorial.

Gómez-Zapiain, J., Ortiz, M. J., \& Gómez-Lope, J. (2012). Capacidad para aportar y solicitar apoyo emocional en las relaciones de pareja en relación con los perfiles de apego. Anales de Psicología, 28(1), 302-312.

Gonsiorek, J. C. (1995). Gay male identities: Concepts and issues. En A. R. D'Augelli \& C. J. Patterson (Eds.), Lesbian, gay, and bisexual identities over the lifespan: Psychological perspectives (pp. 24-47). Nueva York, NY: Oxford University Press.

González, A., Padilla, J. L., Hidalgo, M. D., Gómez-Benito, J., \& Benítez, I. (2011). EASY-DIF: Software for analyzing differential item functioning using the Mantel- 
Haenszel and standardization procedures. Applied Psychological Measurement, 35(6), 483-484. doi:10.1177/0146621610381489

Griffin, D. W., \& Bartholomew, K. (1994). The metaphysics of measurement: The case of adult attachment. En K. Bartholomew \& D. Perlman (Eds.), Advances in personal relationships: Attachment processes in adulthood (Vol. 5, pp. 17-52). Londres: Jessica Kingsley.

Guzmán-González, M., Barrientos, J., Cárdenas, M., Espinoza, M. F., Quijada, P., Rivera, C., ...Tapia, P. (2016). Romantic attachment and life satisfaction in a sample of gay men and lesbians in Chile. International Journal of Sexual Health, 28(2), 141-150. doi:10.1080/19317611.2016.1153014

Guzman, J., Rovella, A., \& Rivarola, M. (2012). La relación de apego y bienestar psicológico en adultos. Revista Diálogos, 3(2), 87-94.

Hazan, C., \& Shaver, P. (1987). Romantic love conceptualized as an attachment process. Journal of Personality and Social Psychology, 52(3), 511-524. doi:10.1037/0022-3514.52.3.511

Hocking, E. C., Simons, R. M., Simons, J. S., \& Freeman, H. (2018). Adult attachment and drinking context as predictors of alcohol problems and relationship satisfaction in college students. The American Journal of Drug and Alcohol Abuse, 44(3), 339-347. doi:10.1080/00952990.2017.1344682

Hu, L., \& Bentler, P. M. (1999). Cutoff criteria for fit indexes in covariance structure analysis: Conventional criteria versus new alternatives. Structural Equation Modeling: A Multidisciplinary Journal, 6(1), 1-55. doi:10.1080/10705519909540118

Jellison, W. A., \& McConnell, A. R. (2004). The mediating effects of attitudes toward homosexuality between secure attachment and disclosure outcomes among gay men. Journal of Homosexuality, 46(1-2), 159-177. doi:10.1300/j082v46n01_05

Katz, J., \& Schneider, M. E. (2015). (Hetero)sexual compliance with unwanted casual sex: Associations with feelings about first sex and sexual self-perceptions. Sex Roles, 72(9-10), 451-461. doi:10.1007/s11199-015-0467-z
Kelley, K. (2018). MBESS: The MBESS R Package. $\mathrm{R}$ package versión 4.4.3. Recuperado de https://CRAN.Rproject.org $/$ package $=$ MBESS

Kinsey, A. C., Pomeroy, W. B., \& Martin, C. E. (1998). Sexual behavior in the human male. Indiana: Indiana University Press (Orig. 1948).

Lafontaine, M. F., Brassard, A., Lussier, Y., Valois, P., Shaver, P. R., \& Johnson, S. M. (2016). Selecting the best items for a shortform of the Experiences in Close Relationships Questionnaire. European Journal of Psychological Assessment, 32(2), 140-154. doi:10.1027/1015-5759/a000243

Li, T., \& Chan, D. K. S. (2012). How anxious and avoidant attachment affect romantic relationship quality differently: A metaanalytic review. European Journal of Social Psychology, 42(4), 406-419. doi:10.1002/ejsp.1842

McDonald, R. P. (1999). Test theory: A unified treatment. Mahwah, NJ: Lawrence Erlbaum.

MacIntosh, H., Reissing, E. D., \& Andruff, H. (2010). Same-sex marriage in Canada: The impact of legal marriage on the first cohort of gay and lesbian Canadians to wed. The Canadian Journal of Human Sexuality, 19(3), 79-90.

Mantel, N. (1963). Chi-square tests with one degree of freedom; extensions of the MantelHaenszel procedure. Journal of the American Statistical Association, 58(303), 690-700. doi:10.1080/01621459.1963.10500879

Mark, K. P., Vowels, L. M., \& Murray, S. H. (2018). The impact of attachment style on sexual satisfaction and sexual desire in a sexually diverse sample. Journal of Sex \& Marital Therapy, 44(5), 450-458. doi:10.1080/0092623x.2017.1405310

Martínez, C., \& Santelices, M. P. (2005). Evaluación del apego en el adulto: Una revisión. Psykhe, 14(1), 181-191. doi:10.4067/S0718-222820050001000280

McLaren, S. (2015). Gender, age, and place of residence as moderators of the internalized homophobia-depressive symptoms relation among Australian gay men and lesbians. Journal of Homosexuality, 62(4), 463-480. doi:10.1080/00918369.2014.983376 
Mikulincer, M. (2006). Attachment, caregiving, and sex within romantic relationships: A behavioral systems perspective. En M. Mikulincer \& G. S. Goodman (Eds.), Dynamics of romantic love: Attachment, caregiving, and sex (pp. 23-44). Nueva York, NY: Guilford Press.

Mikulincer, M., \& Shaver, P. R. (2016). Attachment in adulthood: Structure, dynamics, and change ( $2^{\mathrm{a}}$ ed.). Nueva York, NY: Guilford Press.

Milfont, T. L., \& Fischer, R. (2010). Testing measurement invariance across groups: Applications in cross-cultural research. International Journal of Psychological Research, 3(1), 111-121. doi: $10.21500 / 20112084.857$

Mohr, J. J., \& Fassinger, R. E. (2003). Selfacceptance and self-disclosure of sexual orientation in lesbian, gay and bisexual adults: An attachment perspective. Journal of Counseling Psychology, 50(4), 482-495. doi:10.1037/0022-0167.50.4.482

Pepping, C. A., \& Halford, W. K. (2012). Attachment and relationship satisfaction in expectant first-time parents: The mediating role of relationship enhancing behaviors. Journal of Research in Personality, 46(6), 770-774. doi:10.1016/j.jrp.2012.08.005

Peters, G. (2018). Userfriendlyscience: Quantitative analysis made accessible. R package versión 0.7.2. Recuperado de http://userfriendlyscience.com

R Core Team. (2016). R: A language and environment for statistical computing [Programa informático]. Viena: R Foundation for Statistical Computing. Recuperado de https://www.R-project.org/

Ridge, S. R. \& Feeney, J. A. (1998). Relationship history and relationship attitudes in gay males and lesbians: Attachment style and gender differences. Australian \& New Zealand Journal of Psychiatry, 32(6), 848-859. doi:10.3109/00048679809073875

Riggs, S. A., Cusimano, A. M., \& Benson, K. M. (2011). Childhood emotional abuse and attachment processes in the dyadic adjustment of dating couples. Journal of Counseling Psychology, 58(1), 126-138. doi:10.1037/a0021319
Román, M., Hodges, J., Palacios, J., Moreno, C., \& Hillman, S. (2018). Evaluación de las representaciones mentales de apego a través de las historias incompletas: Aplicación Española de Story Stem Assessment Profile (SSAP). Revista Iberoamericana de Diagnóstico y Evaluación - e Avaliação Psicológica, $\quad$ 1(46), 19 . doi:10.21865/RIDEP46.1.01

RStudio Team. (2018). RStudio: Integrated Development for R [Programa informático]. Boston, MA: RStudio Inc. Recuperado de http://www.rstudio.com/

Sánchez-Fuentes, M. M., Moyano, N., Granados, R., \& Sierra, J. C. (2019). Validation of the Spanish version of the Arizona Sexual Experience Scale (ASEX) using self-reported and psychophysiological measures. Revista Iberoamericana de Psicología y Salud, 10(1), 1-14. doi:10.23923/j.rips.2018.02.021

Sánchez-Fuentes, M. M., Santos-Iglesias, P., Byers, E. S., \& Sierra, J. C. (2015). Validation of the Interpersonal Exchange Model of Sexual Satisfaction Questionnaire in a Spanish sample. The Journal of Sex Research, 52(9), 1028-1041. doi:10.1080/00224499.2014.989307

Santos-Iglesias, P., Vallejo-Medina, P., \& Sierra, J. C. (2009). Propiedades psicométricas de una versión breve de la Escala de Ajuste Diádico en muestras españolas. International Journal of Clinical and Health Psychology, 9(3), 501-517.

Schachner, D. A., \& Shaver, P. R. (2004). Attachment dimensions and sexual motives. Personal Relationships, 11(2), 179-195. doi:10.1111/j.1475-6811.2004.00077.x

Scharfe, E. (2017). Sex differences in attachment. En T. K. Shackelford \& V. A. Shackelford (Eds.), Encyclopedia of Evolutionary Psychological Science (pp 1-5). Nueva York, NY: Springer International.

Sevilla Rodríguez, A. J., Aparicio García, M. E., \& Limiñana Gras, R. M. (2019). La salud de adolescentes y adultos transgénero: Revisión sistemática desde la perspectiva de género. Revista Iberoamericana de Diagnóstico y Evaluación - e Avaliação Psicológica, 1(50), 5-20. doi:10.21865/RIDEP50.1.01 
Sherry, A. (2007). Internalized homophobia and adult attachment: Implications for clinical practice. Psychotherapy: Theory, Research, Practice, Training, 44(2), 219-225. doi:10.1037/0033-3204.44.2.219

Sibley, C. G., \& Liu, J. H. (2004). Short-term temporal stability and factor structure of the revised Experiences in Close Relationships (ECR-R) measure of adult attachment. Personality and Individual Differences, 36(4), 969-975. doi:10.1016/S0191-8869(03)00165-X

Sierra, J. C., Moyano, N., Vallejo-Medina, P., \& Gómez-Berrocal, C. (2018). An abridged Spanish version of Sexual Double Standard Scale: Factorial structure, reliability and validity evidence. International Journal of Clinical and Health Psychology, 18(1), 69-80. doi:10.1016/j.ijchp.2017.05.003

Simon, W., \& Gagnon, J. H. (1986). Sexual scripts: Permanence and change. Archives of Sexual Behavior, 15(2), 97-120. doi: 10.1007/BF01542219

Sperling, M. B., \& Berman, W. H. (Eds.) (1994). Attachment in adults: Clinical and developmental perspectives. Nueva York, NY: Guilford Press.

Starks, T. J., \& Parsons, J. T. (2014). Adult attachment among partnered gay men: Patterns and associations with sexual relationship quality. Archives of Sexual Behavior, 43(1), 107-117. doi:10.1007/s10508-013-0224-8

Ventura-León, J. (2018). Intervalos de confianza para coeficientes omega: propuesta para el cálculo. Adicciones, 30(1), 77-78. doi:10.20882/adicciones.962

Wang, C-C. D. C., Schale, C. L., \& Broz, K. K. (2010). Adult attachment; lesbian, gay, and bisexual identity; and sexual attitudes of nonheterosexual individuals. Journal of College Counseling, 13(1), 31-49. doi:10.1002/j.2161-1882.2010.tb00046.x

Wang, J., \& Wang, X. (2012). Structural equation modeling: Applications using Mplus. Chichester: Higher Education Press.

Wei, M., Russell, D. W., Mallinckrodt, B., \& Vogel, D. L. (2007). The Experiences in Close Relationship Scale (ECR)-Short Form: Reliability, validity, and factor structure.
Journal of Personality Assessment, 88(2), 187-204. doi:10.1080/00223890701268041

Yárnoz-Yaben, S., \& Comino, P. (2011). Evaluación del apego adulto: Análisis de la convergencia entre diferentes instrumentos. Acción Psicológica, 8(2), 67-85. doi:10.5944/ap.8.2.191

Zinbarg, R. E., Revelle, W., \& Yovel, I. (2007). Estimating $\omega \mathrm{h}$ for structures containing two group factors: Perils and prospects. Applied Psychological Measurement, 31(2), 135-157. doi:10.1177/0146621606291558

Zumbo, B. D., Gadermann, A. M., \& Zeisser, C. (2007). Ordinal versions of coefficients alpha and theta for Likert rating scales. Journal of Modern Applied Statistical Methods, 6(1), 2129. doi:10.22237/jmasm/1177992180

Zwick, R., Donoghue, J., \& Grima, A. (1993). Assessment of differential item functioning for performance tasks. Journal of Educational Measurement, 30(3), 233-251. doi:10.1111/j.1745-3984.1993.tb00425.x 